\title{
New insights into innate immune mechanisms underlying allergenicity
}

\author{
M Wills-Karp ${ }^{1}$, A Nathan ${ }^{2}$, K Page $^{3}$ and CL Karp ${ }^{4}$
}

\begin{abstract}
Allergic diseases, which have reached epidemic proportions, are caused by inappropriate immune responses to a relatively small number of environmental proteins. The molecular basis for the propensity of specific proteins to promote maladaptive, allergic responses has been difficult to define. Recent data suggest that the ability of such proteins to promote allergic responses in susceptible hosts is a function of their ability to interact with diverse pathways of innate immune recognition and activation at mucosal surfaces. This review highlights recent insights into innate immune activation by allergens - through proteolytic activity, engagement of pattern recognition receptors, molecular mimicry of TLR signaling complex molecules, lipid-binding activity, and oxidant potential-and the role of such activation in inducing allergic disease. A greater understanding of the fundamental origins of allergenicity should help define new preventive and therapeutic targets in allergic disease.
\end{abstract}

\section{INTRODUCTION}

The prevalence of allergic diseases has increased dramatically over the past few decades, with population prevalence rates reaching $30 \%$ in the industrialized nations. The defining feature of allergic disorders is their association with aberrant levels, and targets, of immunoglobulin E (IgE) production. Allergy is thought to result from maladaptive immune responses to ubiquitous, otherwise innocuous environmental proteins, referred to as allergens. ${ }^{1}$ Allergens, by definition, are proteins that have the ability to elicit powerful T helper lymphocyte type 2 (Th2) responses, culminating in IgE antibody production (atopy). Although allergens represent a minute fraction of the proteins that humans are routinely exposed to, allergenicity is a very public phenomenon, with the identical proteins functioning as allergens in allergic patients. Why specific proteins cause such aberrant $\mathrm{T}$ - and B-cell responses is a basic mechanistic question that has remained largely unanswered.

Allergens derive from a variety of environmental sources, such as plants (trees, grasses), fungi (Alternaria alternata), arthropods (mites, cockroaches), and other mammals (cats, dogs, cows). Allergens constitute a diverse range of molecules, varying in size from small to large multidomain proteins. As they are derived from complex living organisms, they serve a broad range of functions in their respective hosts, from structural to enzymatic. For example, the common house dust mite allergens include several cysteine proteases (Der p 1, Der p 3), serine proteases (Der p 3, Der p 6, Der p 9), chitinases (Der p 15, Der p 18), lipid-binding molecules (Der p 2), and structural molecules such as tropomyosin (Der p 10). Some are species specific; others are molecules with broad biochemical homology that are found in many species. Much work has centered on the study of the allergen epitopes recognized by $\mathrm{T}$ and $\mathrm{B}$ cells. However, as there is no compelling evidence for common structural characteristics among the diverse $\mathrm{T}$ - and $\mathrm{B}$-cell epitopes recognized in allergic responses, ${ }^{2-4}$ it appears doubtful that the presence of such B- and T-cell epitopes are sufficient to endow a protein with allergenic potential. Other factors, such as the size, glycosylation status, resistance to proteolysis, and enzymatic activity, have been suggested to be important in allergenicity. However, none of these factors has been consistently linked with allergenic potential. For example, glycosylation appears not to be a common critical determinant of allergenicity as both glycosylated and nonglycosylated proteins function as food allergens. It may be that there are many structural paths to allergenicity, but the absence of any common structural motif or conformational sequence pattern leaves open the possibility that proteins with

\footnotetext{
${ }^{1}$ Division of Immunobiology, Department of Pediatrics, Cincinnati Children's Hospital Medical Center, University of Cincinnati, Cincinnati, Ohio, USA. ²Division of Neonatology, Department of Pediatrics, Cincinnati Children's Hospital Medical Center, University of Cincinnati, Cincinnati, Ohio, USA. ${ }^{3}$ Division of Critical Care Medicine,

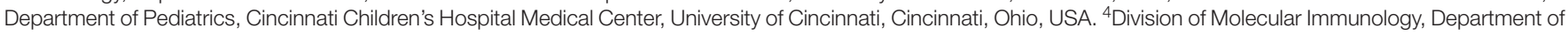
Pediatrics, Cincinnati Children’s Hospital Medical Center, University of Cincinnati, Cincinnati, Ohio, USA. Correspondence: M Wills-Karp (wildc7@cchmc.org) 
allergic potential exhibit a necessary commonality of biological function. Indeed, it has been recently proposed that allergens are linked by their ability to activate the innate immune system of mucosal surfaces, triggering an initial influx of innate immune cells that subsequently promote Th2-polarized adaptive immune responses. It should be noted that, reductive experimental systems aside, natural exposure is not to single, purified proteins, but to complex mixtures of molecules. It may well be that the innate immune-activating molecules are not identical to the proteins recognized by allergic responses, although this would still beg the question as to why those particular proteins are so recognized among the many present during exposure. In this review, we address recent advances in our understanding of the diverse innate immune-activating properties of allergens that appear to endow them with a propensity for promoting Th2 immune responses.

\section{PROTEASE ACTIVITY AND ALLERGIC SENSITIZATION}

Several allergens have cysteine or serine protease activity, including diverse allergens from arthopods (e.g., house dust mites, ${ }^{5-7}$ German cockroaches, ${ }^{8}$ fungi (A. alternata), ${ }^{9}$ and Cladosporium herbarum,${ }^{10}$ mammals (e.g., Felis domesticus) ${ }^{11}$ plants (e.g., pollens from ragweed ${ }^{12}$ ). In addition, many forms of occupational allergy are associated with encounters with proteolytic enzymes such as those used in the manufacture of detergents (alkaline detergents), ${ }^{13}$ or in the food industry (papain). ${ }^{14}$

Several lines of evidence suggest that proteases may facilitate allergen sensitization. First, intrinsic protease activity appears to be linked with sensitization ability in several allergens. Removal of proteases from Aspergillus fumigatus, ${ }^{15}$ German cockroach frass, ${ }^{16}$ American cockroach Per a 10 antigen, ${ }^{17}$ Epi p1 antigen from the fungus Epicoccum purpurascens, ${ }^{18}$ or Cur 11 antigen from the mold Curvularia lunata ${ }^{19}$ was reported to decrease airway inflammation and airway hyperresponsiveness in mouse models of allergic asthma. Second, direct exposure of mice to proteolytic enzymes such as papain can induce allergic sensitization. ${ }^{20}$ Moreover, co-administration of active proteases from A. fumigatus with the tolerogenic antigen, ovalbumin (OVA), resulted in allergic sensitization. ${ }^{15}$ As co-exposure to a tolergenic protein with a protease can induce allergic sensitization, proteases found in ambient air derived from bacterial and viral species may have accessory roles. Finally, subcutaneous injection of a serine protease inhibitor, nafamostat mesilate, during sensitization to house dust mite extracts blunted the development of allergic inflammation and airway hyperresponsiveness. ${ }^{21}$

The exact mechanisms by which proteases can promote allergic sensitization are not well understood. Several mechanisms have been postulated. First, protease activity may increase transepithelial access of allergens to critical cells of the innate immune response, such as dendritic cells (DCs). For example, the cysteine protease Der $\mathrm{p} 1$ can alter epithelial permeability through disruption of epithelial tight junctions and a reduction in ZO-1 and occludin content. ${ }^{6}$ Consistent with this, proteolytic enzymes from a number of tree and grass pollens have also been shown to degrade ZO-1 and disrupt tight junctions. ${ }^{22}$ Moreover, Der $\mathrm{p} 1$ has been shown to cleave $\alpha$-1-anti-trypsin, inhibiting its ability to protect the respiratory tract against serine proteases such as Der $\mathrm{p} 3$ and Der p 9. This may disrupt the proteaseantiprotease balance in mucosal tissues, enhancing the activity of both endogenous and exogenous proteases and leading to enhanced tissue damage and immune activation.

Second, the cysteine protease activity of several mite allergens (Der p 1, Der f 1) may directly impair innate defense mechanisms in the lung by degrading and inactivating lung surfactant proteins (SPs)-A and -D. ${ }^{23}$ SP-A and SP-D are calcium-dependent carbohydrate-binding proteins with multiple innate immune functions, including bacterial agglutination and modulation of leukocyte functions. Importantly, SP-D and SP-A have been shown to protect against allergic inflammation induced by A. fumigatus in mice, ${ }^{24,25}$ likely through binding to glucan moieties of inhaled allergens and facilitation of their clearance.

Proteases may have more direct immunomodulatory actions as well. Proteases from mites, cockroaches, and fungi can increase the expression of cytokines, including interleukin (IL)-6, IL-8, and granulocyte monocyte colony-stimulating factor, ${ }^{5,6,8,9}$ which may lead to the recruitment, activation, and/or enhanced survival of DCs at the mucosal surface. In addition, Der $\mathrm{p} 1$ has been shown to influence the expression of co-stimulatory molecules such as CD40 on DCs. Der p 1 can cleave CD40 on human monocyte-derived DCs, resulting in inhibition of the production of the pivotal Th1-differentiating cytokine, IL-12. ${ }^{26}$ The suppression of CD40 signaling and IL-12 production may induce a shift toward Th2 responses. A similar effect has been observed with the mold Aspergillus (Asp). Exposure of healthy human monocyte-derived DCs to Asp induced their maturation and enhanced their ability to prime Th2 immune responses in allogeneic naive $\mathrm{T}$ cells as compared with naive $\mathrm{T}$ cells primed with lipopolysaccharide (LPS)-activated DCs. ${ }^{27}$ When the proteolytic activity of Asp was neutralized by chemical inactivation, Asp failed to upregulate co-stimulatory molecules on DCs, and these DCs did not prime a Th2 response in naive $\mathrm{T}$ cells. The skewed Th2 response was thought to occur as a result of suppressed IL-12 production by Asp-primed DCs. Interestingly, although the exact mechanisms by which allergen-derived proteases influence the decision-making capability of DCs are not well understood, recent reports suggest that protease containing allergens such as Der $\mathrm{p} 1$ can target two C-type lectins, DC-SIGN and DC-SIGNR. ${ }^{28}$ Loss of DC-SIGN expression after Der $\mathrm{p} 1$ treatment led to a reduction in its binding to its ligand, ICAM-3, on naive T cells, which is thought to be important in Th1 signaling. ${ }^{28}$ Thus, the combined proteolytic activities of Der p 1 on surface expression of molecules, such as CD40 and DC-SIGN, could have profound effects on the decision-making capability of DCs, biasing adaptive immunes response toward a Th2 pattern of response.

Recently, study of the occupational allergen, papain (commonly used in the food industry), has led to the novel hypothesis that proteases can directly prime $\mathrm{Th} 2 \mathrm{immune}$ responses through actions on basophils. ${ }^{20}$ Data suggest that papain can cleave a yet-to-be identified host sensor which, in turn, activates basophils to produce IL-4, and thymic stromal lymphopoietinpromoting Th2 differentiation. This intriguing work supports 


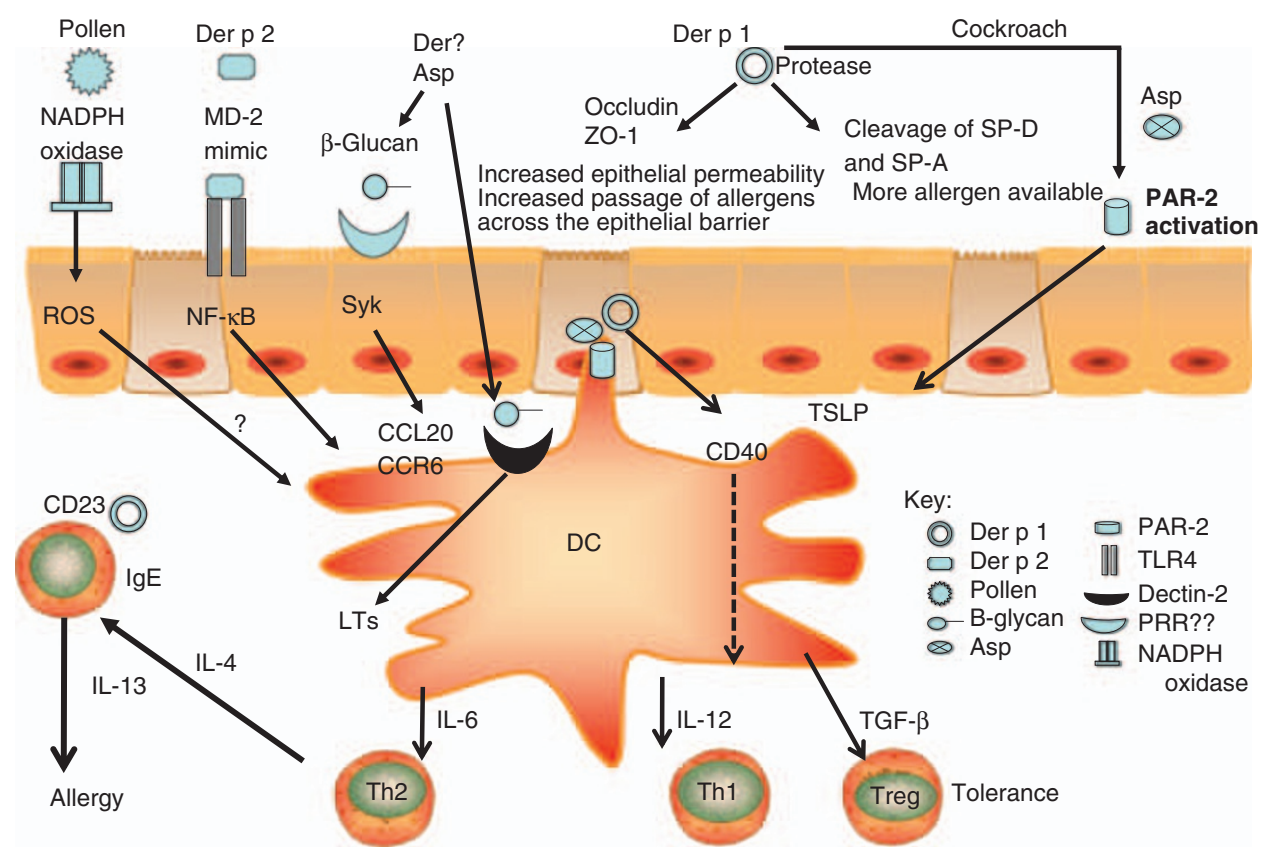

Figure 1 Schematic of innate immune mechanisms activated by allergens.

the concept that host detection of protease activity associated with allergens may provide a unique pathway of innate immune activation. The generality of this pathway, as well as its molecular identification, remains to be defined.

Allergen-derived proteases can have direct effects on adaptive immune responses as well, through cleavage of molecules such as CD25 and CD23. Specifically, Der p 1 has been shown to be able to cleave the $\alpha$-chain of the IL-2 receptor (CD25) on human $\mathrm{T}$ cells ${ }^{29}$ (Figure 1). As a result, $\mathrm{T}$ cells exposed directly to Der $\mathrm{p} 1$ show markedly reduced Th1 cytokine production and enhanced Th2 cytokine production, something dependent on the protease activity or Der p 1. Cleavage of CD25 might also, of course, alter regulatory function, as IL-2 stimulation is required for the maintenance of regulatory $\mathrm{T}$ cells in the periphery. The overall effect may be to shift the balance of immune responses from a tolerogenic response to one favoring a Th2 pattern of response. Allergenic proteases such as Der $\mathrm{p} 1$ have also been shown to be able to cleave the low-affinity receptor for IgE, CD23, from the surface of human B cells, releasing the soluble form of the receptor ${ }^{30}$ (Figure 1). As the membrane-bound form of the IgE receptor is thought to exert its effect as a negative regulator of IgE synthesis, Der $p 1$ cleavage of CD23 could potentially disrupt the negative feedback signal and enhance IgE synthesis, thereby amplifying the allergic response. Antitrypsin can inhibit this effect of Der p 1 on CD23 cleavage, suggesting that disruption of the balance between proteases and protease inhibitors might have a role in allergic sensitization. It should be noted that whether intact proteases such as Der $\mathrm{p} 1$ actually gain functional access to lymphocytes in vivo remains an open question.

The biological effects of some allergenic proteases may also be mediated through activation of the protease-activated receptor 2 (PAR2). PARs $(1,2,3,4)$ are a family of proteolytically activated
G-protein-coupled receptors. Proteases cleave within the $\mathrm{N}$ terminus of the receptors and expose a tethered ligand domain that binds and activates the cleaved receptor. Several lines of evidence suggest that PAR2, in particular, may be important in allergic sensitization. It is expressed by many cells in the lung, including airway epithelial cells, ${ }^{31}$ fibroblasts, ${ }^{32}$ macrophages ${ }^{33}$ and mast cells, ${ }^{34}$ and, importantly, patients with asthma have been shown to exhibit increased expression of PAR2 on respiratory epithelial cells. ${ }^{35}$ Several house dust mite allergens (Der p1, Der p 3, Der p 9), along with German cockroach extract, ${ }^{36}$ have been shown to be able to cleave and activate PAR2 (Figure 1). Several reports have shown that activation of PAR2 by house dust mite extract, ${ }^{7}$ German cockroach, ${ }^{37}$ or the mold allergen Pen c $13^{38}$ leads to increased cytokine production by airway epithelia. Specifically, airway epithelial cells were shown to increase the expression of thymic stromal lymphopoietin through the activation of PAR2 when treated with papain, trypsin, or the fungus Alterneria. ${ }^{9}$ As thymic stromal lymphopoietin promotes DC polarization of naive $\mathrm{T}$ cells to a Th2 phenotype, these results suggest that PAR2 activation may serve as a link between innate and adaptive immune responses.

Several mouse models of allergic inflammation have also underscored a potential role for PAR2 in allergic sensitization. For example, one recent study showed that tolerance to inhaled OVA could be overcome by co-administration of PAR2-activating peptides to the airways, promoting allergic sensitization. ${ }^{39}$ Other studies have shown that overexpression of PAR2 in mice renders them susceptible to allergic airway inflammation when sensitized and challenged locally with OVA, as compared with wild-type controls. ${ }^{40}$ In contrast, PAR2-deficient mice were protected against allergic inflammation. Although these data strongly support the concept that protease activity may lead to airway allergic sensitization through PAR2 activation, there are 
a few reports suggesting that PAR2 activation may also reduce airway inflammation. For example, despite the fact that TLR4 (vide infra) and PAR2 signaling have been shown to exhibit cooperativity, ${ }^{41}$ PAR2-activating peptides have been reported to inhibit LPS-induced neutrophil influx into mouse airways. ${ }^{42}$ In a rabbit model of experimental asthma, sensitization to the pollen Parietaria judaica, followed by allergen challenge in the presence or absence of a PAR2-activating peptide, led to PAR2-mediated attenuation of the development of airway hyperresponsiveness and airway eosinophilia. ${ }^{43}$ It is possible that the discrepancies in these results are due to the timing of PAR2 activation, with activation by exogenous proteases during generation of immune responses having different effects than that occurring in the midst of an ongoing inflammatory response.

\section{TOLL-LIKE RECEPTORS, LIPID-BINDING ACTIVITY, AND ALLERGIC SENSITIZATION}

Recent recognition of the critical roles of innate pattern recognition receptors such as Toll-like receptors, NOD-like receptors, RIG-I-like receptors, and C-type lectin receptors, in activation and instruction of antigen-presenting cells ${ }^{44}$ has led to the exploration of the role of these pathways in allergic responses. It will be noted that, although TLR-promoted activation of Th1 responses by DCs is well studied and well understood, the receptors and pathways promoting Th2 immune responses have been considerably less tractable to experimental investigation.

Numerous studies have probed the role of TLR4 signaling in allergic inflammation. Epidemiological studies have reported an inverse correlation between high levels of bacterial products, such as LPS in the ambient environment during very early life and the subsequent development of atopy and allergic disease. ${ }^{45-47}$ It has been postulated, pace the hygiene hypothesis, that such exposures mediate robust counter-regulatory tone in the developing immune system. ${ }^{48}$ LPS exposure can also exacerbate established asthma, however, probably by direct stimulation of airway pro-inflammatory responses. ${ }^{49}$ Experimental mouse models have provided mechanistic insight into the ability of LPS exposure to regulate the development of allergic asthma. As predicted by the hygiene hypothesis, LPS dose appears to be a critical variable. Although airway sensitization with OVA along with "very low dose" ( $<1$ ng) LPS was reported to induce tolerance, sensitization in the presence of "low-dose" (100 ng) LPS promoted TLR-dependent, Th2 inflammation, and sensitization in the presence of "high-dose" $(100 \mu \mathrm{g})$ LPS led to a Th1 (and likely a regulatory) response. ${ }^{50,51}$ Recent studies using bone marrow chimeras indicate that TLR4 signaling in radioresistant cells, not radiosensitive hematopoietic cells, is necessary and sufficient for DC activation and priming of allergic effector $\mathrm{T}$ helper responses in the lung in response to dust mite extracts. ${ }^{52}$ As for other Toll-like receptors, TLR2 ligands have been shown to be able to promote ${ }^{53}$ or inhibit ${ }^{54} \mathrm{Th} 2$ differentiation and allergic inflammation in the lung.

A recent study has reported a more direct link between TLR signaling and allergic sensitization. Among defined dust mite antigens, Der p 2 and Der $\mathrm{f} 2$ have the highest rates of skin test positivity in atopic patients. ${ }^{55}$ Notably, sequence homology places these allergens in the MD-2-related lipid-recognition domain family of proteins ${ }^{56,57}-\mathrm{MD}-2$ being a secreted protein that is the LPS-binding member of TLR4 signaling complex. As the crystal structures of Der $\mathrm{p} 2$ and MD-2 exhibit structural homology, Trompette et al. ${ }^{58}$ examined whether Der $\mathrm{p} 2$ exhibited functional homology as well. Indeed, they reported that Der p 2 can facilitate TLR4 signaling through direct interactions with the TLR4 complex, reconstituting LPS-promoted TLR4 signaling in the absence of MD-2 and facilitating such signaling in the presence of MD-2 (Figure 1). They further found that Der p 2 could facilitate LPS signaling in primary antigen-presenting cells, with or without MD-2 being present. Finally, they reported that the in vitro functional and biochemical activities of Der $\mathrm{p}$ 2 show its in vivo allergenicity-Der $\mathrm{p} 2$ promotes experimental allergic asthma in a TLR4-dependent manner, retaining this property in mice with a genetic deletion of MD-2. These data suggest that Der $\mathrm{p}$ 2's propensity to be targeted by the adaptive immune response is a function of its autoadjuvant properties. In the light of this study, it should be noted that efficient generation of effector T-cell responses has been shown to depend on the presence of TLR ligands in the specific DC phagosome that contains the antigen. ${ }^{59}$ In the case of Der $\mathrm{p} 2$, antigen and TLR ligand are, perforce, colocalized. These data also suggest the possibility that Der p 2-mediated facilitation of TLR4 signaling under conditions of bacterial product exposure-those associated with increasing rates of aeroallergy in the urban, Westernized world-may shift the LPS-response curve from the tolerizing into the Th2-inducing range. Der $\mathrm{p} 2$ may also promote exacerbation of established asthma by facilitating TLR4 signaling by airway epithelial cells-cells reported to express TLR4, but little or no MD-2, in the basal state. ${ }^{60}$

Several other members of the MD-2-like lipid-binding family are major allergens, ${ }^{4}$ suggesting generality for these findings. More broadly, however, more than $50 \%$ of defined major allergens are thought to be lipid-binding proteins, ${ }^{4}$ something that suggests that intrinsic adjuvant activity by such proteins and their lipid cargo is likely to have wide generality as a mechanism underlying the phenomenon of allergenicity. Further studies defining the lipids normally bound by these allergens, the receptors thereby activated, and the pathways of innate and adaptive immune response promoted by such activation are awaited. It should be noted that, in addition to activation of Toll-like receptors, lipid ligands are known to be important promoters (and targets) of innate lymphocyte responses.

\section{CARBOHYDRATE STRUCTURES AND ALLERGIC SENSITIZATION}

Recent data also suggest an importance of complex carbohydrates in promoting Th2 immune responses. Helminth-derived carbohydrates such as lacto- $N$-fucopentaose III have been shown to promote Th2 responses through their ability to activate DCs in vivo. ${ }^{61}$ In addition, lacto- $N$-fucopentaose III has been shown to be able to promote Th2 responses to a co-administered, unrelated antigen such as human serum albumin. ${ }^{62}$ Though the mechanism underlying DC activation by lacto- $N$-fucopentaose III glycoconjugates has not been fully elucidated, it involves 
ligation of C-type lectins on the DC, leading to subsequent antagonism of TLR signaling. Support for a broad role for complex carbohydrates, in particular glucans, in allergen-associated Th2 immune responses is emerging. Glucans are a diverse class of naturally occurring glucose polymers, which can be short or long, branched or unbranched, exist as $\alpha$ - or $\beta$-isomers, and be soluble or particulate. For the purpose of this discussion, we are mostly concerned with the $\beta$-glucans, which contain a polyglucose, $(1 \rightarrow 3)-\beta$-D-glucan, and are commonly found in the cell walls of fungi, pollens, and certain bacteria. In plants, polymers of $\beta$-glucans are thought to protect the developing pollen during meiosis, and are later destroyed by the enzyme $(1 \rightarrow 3)-\beta$-D-glucanase to liberate the microspores. Although $\beta$-glucans are widely expressed, they are not found in mammalian cells. As such, they can exert their effect as pathogen-associated molecular patterns, triggering immune responses through activation of specific innate pattern recognition receptors.

The immunostimulatory properties of $\beta$-glucans have been recognized for decades, since their identification as the immunoactive component of mushrooms. ${ }^{63}$ Recently, $\beta$-glucans have been shown to be able to promote Th2 responses. For example, it has been reported that $\beta$-glucan structures present in the peanut glycoallergen Ara h 1 have Th2-inducing characteristics; ${ }^{64}$ native, but not deglycosylated, Ara $\mathrm{h} 1$ has been shown to activate human monocyte-derived DC and induce IL-4- and IL-13secreting Th2 cells.

Exposure to $\beta$-glucans has also been shown to induce airway hyperresponsiveness in allergic humans. ${ }^{65}$ Studies in guinea pigs have shown that direct delivery of $(1 \rightarrow 3)-\beta$-D-glucan to the airways can induce the recruitment of lung eosinophils and lymphocytes. ${ }^{66}$ In mice, exposure to soluble $\beta$-glucan isolated from Candida albicans ${ }^{67}$ markedly exacerbated OVA-induced eosinophilic airway inflammation, concomitant with enhanced lung expression of Th2 cytokines and IL-17A. Exposure to $\beta$-glucans and OVA increased the number of cells bearing major histocompatibility complex class II and the expression of antigenpresenting cell-related molecules such as CD80, CD86, and DEC205 on bone marrow-derived DCs. In support of a role of $\beta$-glucans in the recruitment and activation of DCs at mucosal surfaces, recent studies have shown that $\beta$-glucans contained in house dust mite extracts and in molds may initiate immune responses at the mucosal surface. House dust mite extract-mediated induction of the release of the chemokine, CCL20, which recruits immature DCs, by human airway epithelial cells in culture, was shown to occur through $\beta$-glucan and Syk-dependent signaling pathways ${ }^{68}$ (Figure 1). Although the exact lectin receptor mediating these effects was not identified in these studies, the results suggested that $\beta$-glucan moieties contained in house dust mite extracts might mediate early processes leading to immature DC recruitment to the airways. This concept is supported by another recent study that showed that dectin-2 receptor signaling pathways (Dectin-2/FcR $\gamma /$ Syk) mediated the production of cysteinyl leukotrienes in bone marrow-derived DCs following stimulation with house dust mite extracts or Aspergillus $^{69}$ (Figure 1). Taken together, these findings identify the dectin-2/FcR $\gamma /$ Syk axis as a novel receptor-mediated pathway by which several potent allergens are recognized by innate immune cells at the airway surface, linking them with the development of Th2-skewed adaptive immune responses.

Consistent with a role for lectins in promoting Th2 immune responses, blockade of the mannose receptor, an endocytic C-type lectin receptor, significantly reduced Der $\mathrm{p} 1$ uptake by DCs. ${ }^{70}$ These findings are consistent with previous findings suggesting that engagement of the mannose receptor by selected ligands on human DCs leads to the induction of a DC phenotype favoring Th2 polarization. ${ }^{71}$ Although the study of the role of glucans as Th2-inducing pathogen-associated molecular patterns is only in its infancy, data to date suggest that carbohydrate moieties contained in common allergens function as strong Th2 inducers through activation of variety of C-type lectin receptors on DCs.

\section{OXIDATIVE ACTIVITY AND ALLERGEN SENSITIZATION}

It has been recently shown that common allergenic pollen grains contain nicotinamide adenine dinucleotide phosphate (reduced) oxidase activity as well as allergens. ${ }^{72}$ Such pollen grains have been shown to increase the levels of reactive oxygen species significantly in cultured cells, and to be able to induce allergic airway inflammation in experimental animals $^{73}$ (Figure 1). Pretreatment of these pollen grains with nicotinamide adenine dinucleotide phosphate oxidase inhibitors attenuated their capacity to increase reactive oxygen species levels in airway epithelial cells and subsequent airway inflammation. Similarly, pretreatment of mice with antioxidants has been shown to prevent the development of pollen-mediated asthma in mice. Interestingly, delaying antioxidant treatment until after pollen challenge was ineffective, suggesting that the oxidase activity is of critical importance during the period of innate immune activation. Although the mechanisms remain to be defined, it has been speculated that nicotinamide adenine dinucleotide phosphate oxidase activity initiates immune activation through its ability to recruit inflammatory cells, possibly through the induction of IL- 8 by p38 mitogen-activated protein kinase. ${ }^{74}$ Of interest, genetic polymorphisms in genes regulating oxidative stress have been shown to be associated with susceptibility to asthma in several populations. ${ }^{75}$

\section{CONCLUSIONS}

Although allergens are a diverse group of molecules, it is becoming increasingly clear that their allergenicity likely resides in their ability to activate various innate immune pathways at mucosal surfaces, rather than in any structural similarities. Complex allergens contain multiple innate immune-activating components, which trigger the initial mucosal influx of innate immune cells that subsequently promote Th2-polarized adaptive immune responses. Although the study of innate activating properties of allergens is in its infancy, it is clear that a better molecular understanding of the fundamental origins of allergenicity may well lead to the development of new therapeutic strategies to block allergen recognition and the ensuing inflammatory cascade effectively. 


\section{DISCLOSURE}

The authors declared no conflict of interest.

C) 2010 Society for Mucosal Immunology

\section{REFERENCES}

1. Wills-Karp, M. Immunologic basis of antigen-induced airway hyperresponsiveness. Annu. Rev. Immunol. 17, 255-281 (1999).

2. Traidl-Hoffmann, C., Jakob, T. \& Behrendt, H. Determinants of allergenicity. J. Allergy Clin. Immunol. 123, 558-566 (2009).

3. Aalberse, R.C. Structural biology of allergens. J. Allergy Clin. Immunol. 106, 228-238 (2000).

4. Thomas, W.R., Hales, B.J. \& Smith, W.-A. Structural biology of allergens. Curr. Allergy Asthma Rep. 5, 388-393 (2005).

5. King, C., Brennan, S., Thompson, P.J. \& Stewart, G.A. Dust mite proteolytic allergens induce cytokine release from cultured airway epithelium. J. Immunol. 161, 3645-3651 (1998).

6. Wan, H. et al. Der p1 facilitates transepithelial allergen delivery by disruption of tight junctions. J. Clin. Invest. 104, 123-133 (1999).

7. Sun, G., Stacey, M.A., Schmidt, M., Mori, L. \& Mattoli, S. Interaction of mite allergens Der p3 and Der p9 with protease-activated receptor-2 expressed by lung epithelial cells. J. Immunol. 167, 1014-1021 (2001).

8. Bhat, R.K., Page, K., Tan, A. \& Hershenson, M.B. German cockroach extract increases bronchial epithelial cell interleukin-8 expression. Clin. Exp. Allergy 33, 35-42 (2003).

9. Kouzaki, H., O'Grady, S.M., Lawrence, C.B. \& Kita, H. Proteases induce production of thymic stromal lymphopoietin by airway epithelial cells through protease-activated receptor-2. J. Immunol. 183, 1427-1434 (2009).

10. Kauffman, H.F., Tamm, M., Timmerman, J.A.B. \& Borger, P. House dust mite major allergens Der $\mathrm{p} 1$ and Der $\mathrm{p} 5$ activate human airway-derived epithelial cells by protease-dependent and protease-independent mechanisms. Clin. Mol. Allergy 4, 5 (2006).

11. Ring, P.C. et al. The $18 \mathrm{kDa}$ form of cat allergen Felis domesticus 1 (Fel d 1) is associated with gelatin- and fibronectin-degrading activity. Clin. Exp. Allergy 30, 1085-1096 (2000).

12. Widmer, F., Hayes, P.J., Whittaker, R.G. \& Kumar, R.K. Substrate preference profiles of proteases released by allergenic pollens. Clin. Exp. Allergy 30, 571-576 (2000).

13. Saeki, K., Ozaki, K., Kobayashi, T. \& Ito, S. Detergent alkaline proteases: enzymatic properties, genes, and crystal structures. J. Biosci. Bioeng. 103, 501-508 (2007).

14. Novey, H.S., Marchioli, L.E., Sokol, W.N. \& Wells, I.D. Papain-induced asthma-physiological and immunological features. J. Allergy Clin. Immunol. 63, 98-103 (1979).

15. Kheradmand, F. et al. A protease activated pathway underlying Th2 cell type activation and allergic lung disease. J. Immunol. 169, 5904-5911 (2002).

16. Page, K. et al. TLR2-mediated activation of neutrophils in response to German cockroach frass. J. Immunol. 180, 6317-6324 (2008).

17. Sudha, V.T., Arora, N. \& Singh, B.P. Serine protease activity of Per a 10 augments allergen-induced airway inflammation in a mouse model. Eur. J. Clin. Invest. 39, 507-516 (2009).

18. Kukreja, N., Sridhara, S., Singh, B.P. \& Arora, N. Effect of proteolytic activity of Epicoccum purpurascens major allergen, Epi p1 in allergic inflammation. Clin. Exp. Immunol. 154, 162-171 (2008).

19. Tripathi, P., Kukreja, N., Singh, B.P. \& Arora, N. Serine protease activity of Cur 11 from Curvularia lunata augments Th2 response in mice. J. Clin. Immunol. 29, 292-302 (2009).

20. Sokol, C.L. et al. Basophils function as antigen-presenting cells for an allergeninduced Thelper type 2 response. Nat. Immunol. 10, 712-720 (2009).

21. Chen, C.L. et al. Serine protease inhibitors nafamostat mesilate and gabexate mesilate attenuate allergen-induced airway inflammation and eosinophilia in a murine model of asthma. J. Allergy Clin. Immunol. 118, 105-122 (2006).

22. Runswick, S., Mitchell, T., Davies, P., Robinson, C. \& Garrod, D.R. Pollen proteolytic enzymes degrade tight junctions. Respirology 12, 834-842 (2007).

23. Shakib, F., Ghaemmaghami, A.M. \& Sewell, H.F. The molecular basis of allergenicity. Trends Immunol. 29, 633-640 (2008).

24. Brandt, E.B. et al. Surfactant protein D alters allergic lung responses in mice and human subjects. J. Allergy Clin. Immunol. 121, 1140-1147 (2008).
25. Madan, T. et al. Surfactant proteins A and D protect mice against pulmonary hypersensitivity induced by Aspergillus fumigatus antigens and allergens. J. Clin. Invest. 207, 467-475 (2001).

26. Ghaemmaghami, A.M., Gough, L., Sewell, H.F. \& Shakib, F. The proteolytic activity of the major dust mite allergen Der $\mathrm{p} 1$ conditions dendritic cells to produce less interleukin-12: allergen-induced Th2 bias determined at the dendritic cell level. Clin. Exp. Allergy 32, 1468-1475 (2002).

27. Lamhamedi-Cherradi, S.E. et al. Fungal proteases induce Th2 polarization through limited dendritic cell maturation and reduced production of IL-12. J. Immunol. 180, 6000-6009 (2008).

28. Furmonaviciene, R. et al. Der $\mathrm{p} 1$ cleaves cell surface DC-SIGN and DC-SIGNR: experimental analysis of in silico substrate identification and implications in allergic responses. Clin. Exp. Allergy 37, 231-242 (2007).

29. Schulz, O. et al. Proteolytic cleavage of CD25, the a subunit of the human T cell interleukin 2 receptor, by Der $\mathrm{p} 1$, a major mite allergen with cysteine protease activity. J. Exp. Med. 187, 271-275 (1998).

30. Schulz, O. et al. Der p 1, a major allergen of the house dust mite, proteolytically cleaves the low-affinity receptor for human lgE (CD23). Eur. J. Immunol. 25, 3191-3194 (1995).

31. Asokananthan, N. et al. Activation of protease-activated receptor (PAR)-1, PAR-2 and PAR-4 stimulates IL-6, IL-8 and prostaglandin E2 release from human respiratory epithelial cells. J. Immunol. 16, 3577-3585 (2002).

32. Akers, I.A. et al. Mast cell tryptase stimulates human lung fibroblast proliferation via protease-activated receptor-2. Am. J. Physiol. 278, 193-201 (2000).

33. Colognato, R. et al. Differential expression and regulation of proteaseactivated receptors in human peripheral monocytes and monocytederived antigen presenting cells. Blood 102, 2645-2652 (2003).

34. D'Andrea, M.R., Rogahn, C.J. \& Andrade-Gordon, P. Localization of protease-activated receptors-1 and -3 in human mast cells; indications for an amplified mast cell degranulation cascade. Biotech. Histochem. 75, 85-90 (2000).

35. Knight, D.A. et al. Protease-activated receptors in human airways: upregulation of PAR-2 in respiratory epithelial cells from patients with asthma. J. Allergy Clin. Immunol. 108, 797-803 (2001).

36. Hong, J.H. et al. German cockroach extract activates protease-activated receptor 2 in human airway epithelial cells. J. Allergy Clin. Immunol. 113, 315-319 (2004).

37. Page, K., Strunk, V.S. \& Hershenson, M.B. Cockroach proteases increase IL-8 expression in human bronchial epithelial cells via activation of protease-activated receptor-2 and extracellular-signal-regulated kinase. J. Allergy Clin. Immunol. 112, 1112-1118 (2003).

38. Chiu, L.L., Perng, D.W., Yu, C.H., Su, S.N. \& Chow, L.P. Mold allergen, Pen c13, induced IL-8 expression in human airway epithelial cells by activated protease-activated receptor 1 and 2. J. Immunol. 178, 52375244 (2007).

39. Ebeling, C. et al. Proteinase-activated receptor 2 activation in the airways enhances antigen-mediated airway inflammation and airway hyperresponsiveness. J. Allergy Clin. Immunol. 115, 623-630 (2005).

40. Schmidlin, F. et al. Protease-activated receptor 2 mediates eosinophil infiltration and hyperreactivity in allergic inflammation of the airway. J. Immunol. 169, 5315-5321 (2002).

41. Rallabhandi, P. et al. Analysis of proteinase-activated receptor 2 and TLR4 signal transduction: a novel paradigm for receptor cooperativity. J. Biol. Chem. 283, 24314-24325 (2008).

42. Moffatt, J.D., Jeffrey, K.L. \& Cocks, T.M. Protease-activated receptor-2 activating peptide SLIGRL inhibits bacterial lipopolysaccharide-induced recruitment of polymorphonuclear leukocytes into the airways of mice. Am. J. Respir. Cell Mol. Biol. 26, 680-684 (2002).

43. D'Augostino, B. et al. Activation of protease-activated receptor-2 reduces airway inflammation in experimental allergic asthma. Clin. Exp. Allergy 37, 1436-1443 (2007).

44. Iwasaki, A. \& Medzhitov, R. Toll-like receptor control of the adaptive immune responses. Nat. Immunol. 5, 987-995 (2004).

45. Braun-Fahrlander, C. et al. Environmental exposure to endotoxin and its relation to asthma in school-age children. N. Engl. J. Med. 347, 869-877 (2002).

46. Gehring, U. et al. House dust endotoxin and allergic sensitization in children. Am. J. Respir. Crit. Care Med. 166, 939-944 (2002).

47. Riedler, J. et al. Exposure to farming in early life and development of asthma and allergy: a cross-sectional survey. Lancet 358, 1129-1133 (2001). 
48. Wills-Karp, M. et al. The germless theory of allergic disease: revisiting the hygiene hypothesis. Nat. Rev. Immunol. 1, 69-75 (2001).

49. Michel, O. et al. Effect of inhaled endotoxin on bronchial reactivity in asthmatic and normal subjects. J. Appl. Physiol. 66, 1059-1064 (1989).

50. Eisenbarth, S.C. et al. Lipopolysaccharide-enhanced, Toll-like receptor 4-dependent T helper cell type 2 responses to inhaled antigen. J. Exp. Med. 196, 1645-1651 (2002).

51. Herrick, C.A. \& Bottomly, K. To respond or not to respond: T cells in allergic asthma. Nat. Rev. Immunol. 3, 405-412 (2003).

52. Hammad, H. et al. House dust mite allergen induces asthma via Toll-like receptor 4 triggering of airway structural cells. Nat. Med. 15, 410-416 (2009).

53. Pulendran, B. et al. Lipopolysaccharides from distinct pathogens induce different classes of immune responses in vivo. J. Immunol. 167, 5067-5076 (2001)

54. Page, K., Ledford, J.R., Zhou, P. \& Wills-Karp, M. A TLR2 agonist in German cockroach frass activates MMP-9 release and is protective against allergic inflammation in mice. J. Immunol. 183, 3400-3408 (2009).

55. Heymann, P.W. et al. Antigenic and structural analysis of group II allergens (Der $\mathrm{fll}$ and Der $\mathrm{p} \mathrm{II)} \mathrm{from} \mathrm{house} \mathrm{dust} \mathrm{mites} \mathrm{(Dermatophagoides} \mathrm{spp).}$ J. Allergy Clin. Immunol. 83, 1055-1067 (1989).

56. Inohara, N. \& Nuñez, G. ML-a conserved domain involved in innate immunity and lipid metabolism. Trends Biochem. Sci. 27, 219-221 (2002).

57. Gruber, A., Mancek, M., Wagner, H., Kirschning, C.J. \& Jerala, R. Structural model of MD-2 and functional role of its basic amino acid clusters involved in cellular lipopolysaccharide recognition. J. Biol. Chem. 279, 28475-28482 (2004).

58. Trompette, A. et al. Allergenicity resulting from functional mimicry of a Tolllike receptor complex protein. Nature 457, 585-588 (2009).

59. Blander, J.M. \& Medzhitov, R. Toll dependent selection of microbial antigens for presentation by dendritic cells. Nature 440, 808-812 (2006).

60. Jia, H.P. et al. Endotoxin responsiveness of human airway epithelia is limited by low expression of MD-2. Am. J. Physiol. Lung Cell Mol. Physiol. 287, L428-L437 (2004).

61. Thomas, P.G., Carter, M.R., Da'Dara, A.A., DeSimone, T.M. \& Harn, D.A. A helminth glycan induces APC maturation via alternative NF-kappa B activation independent of I kappa B alpha degradation. J. Immunol. 175, 2082-2090 (2005).

62. Okano, M., Satoskar, A.R., Nishizaki, K. \& Harn, D.A. Jr. Lacto-Nfucopentaose III found on Schistosoma mansoni egg antigens functions as adjuvant for proteins by inducing Th2-type response. J. Immunol. 167, 442-450 (2001).
63. Goodridge, H.S., Wolf, A.J. \& Underhill, D.M. $\beta$-Glucan recognition by the innate immune system. Immunol. Rev. 230, 38-50 (2009).

64. Shreffler, W.G. et al. The major glycoprotein allergen from Arachis hypogaea, Ara h 1, is a ligand of dendritic cell-specific ICAM-grabbing non-integrin and acts as a Th2 adjuvant in vitro. J. Immunol. 177, 3677-3685 (2006).

65. Rylander, R. Airways responsiveness and chest symptoms after inhalation of endotoxin or (1-3)- $\beta$-D-glucan. Indoor Built. Environ. 5, 106-111 (1996).

66. Fogelmark, B., Thorn, J. \& Rylander, R. Inhalation of ( $1 \rightarrow 3$ )-beta-D-glucan causes airway eosinophilia. Mediators Inflamm. 10, 13-19 (2001).

67. Inoue, K. et al. Candida soluble cell wall beta-glucan facilitates ovalbumininduced allergic airway inflammation in mice: possible role of antigenpresenting cells. Respir. Res. 10, 68 (2009).

68. Nathan, A.T., Peterson, E.A., Chakir, J. \& Wills-Karp, M. Innate immune responses of airway epithelium to house dust mite are mediated through beta-glucan-dependent pathways. J. Allergy Clin. Immunol. 123, 612-618 (2009).

69. Barrett, N.A., Maekawa, A., Rahman, O.M., Austen, K.F. \& Kanaoka, Y. Dectin-2 recognition of house dust mite triggers cysteinyl leukotriene generation by dendritic cells. J. Immunol. 182, 1119-1128 (2009).

70. Deslée, G. et al. Involvement of the mannose receptor in the uptake of Der p 1, a major mite allergen, by human dendritic cells. J. Allergy Clin. Immunol. 110, 763-770 (2002).

71. Chieppa, M. et al. Cross-linking of the mannose receptor on monocytederived dendritic cells activates an anti-inflammatory immunosuppressive program. J. Immunol. 171, 4552-4560 (2003).

72. Boldogh, I. et al. ROS generated by pollen NADPH oxidase provide a signal that augments antigen-induced allergic airway inflammation. J. Clin. Invest. 115, 2169-2179 (2005).

73. Dharajiya, N., Choudhury, B.K., Bacsi, A., Boldogh, I., Alam, R. \& Sur, S. Inhibiting pollen reduced nicotinamide adenine dinucleotide phosphate oxidase-induced signal by intrapulmonary administration of antioxidants blocks allergic airway inflammation. J. Allergy Clin. Immunol. 119, 646-653 (2007).

74. Bacsi, A., Dharajiya, N., Choudhury, B.K., Sur, S. \& Boldogh, I. Effect of pollen-mediated oxidative stress on immediate hypersensitivity reactions and late-phase inflammation in allergic conjunctivitis. J. Allergy Clin. Immunol. 116, 836-843 (2005).

75. Islam, T. et al. Glutathione-S-transferase (GST) P1, GSTM1, exercise, ozone and asthma incidence in school children. Thorax 64, 197-202 (2009). 